\title{
Genome-wide association study of clinical parameter in immunoglobulin light chain amyloidosis in three patient cohorts
}

Iman Meziane ${ }^{1}$, Miguel Inacio da Silva Filho ${ }^{1}$, Niels Weinhold ${ }^{3,4}$, Chiara Campo ${ }^{1}$, Stefanie Huhn ${ }^{3}$, Jolanta Nickel $^{3}$, Per Hoffmann ${ }^{5,6}$, Markus M Nöthen ${ }^{5,7}$, Karl-Heinz Jöckel ${ }^{8}$, Stefano Landi ${ }^{9}$, Jonathan S. Mitchell ${ }^{10}$, David Johnson ${ }^{11}$, Anna Jauch ${ }^{12}$, Gareth J. Morgan ${ }^{4}$, Richard Houlston ${ }^{10,11}$, Hartmut Goldschmidt ${ }^{3,13}$, Paolo Milani $^{14}$, Giampaolo Merlini ${ }^{14}$, Dorota Rowcieno ${ }^{15}$, Philip Hawkins ${ }^{15}$, Ute Hegenbart ${ }^{3}$, Giovanni Palladini ${ }^{14}$, Ashutosh Wechalekar ${ }^{15}$, Asta Försti ${ }^{1,2 *}$, Stefan O. Schönland ${ }^{3 *}$, Kari Hemminki $^{1,2 *}$. * = shared last authorship

1. Division of Molecular Genetic Epidemiology, German Cancer Research Center (DKFZ), Heidelberg, Germany

2. Center for Primary Health Care Research, Lund University, Malmo, Sweden

3. Department of Internal Medicine V, University of Heidelberg, Heidelberg, Germany

4. Myeloma Institute, University of Arkansas for Medical Sciences, Little Rock, AR, USA.

5. Institute of Human Genetics, University of Bonn, Bonn, Germany

6. Department of Biomedicine, University of Basel, Basel, Switzerland

7. Department of Genomics, Life \& Brain Research Center, University of Bonn, Bonn, Germany

8. Institute for Medical Informatics, Biometry and Epidemiology, University Hospital Essen, University of Duisburg-Essen, Germany

9. Department of Biology, University of Pisa, Pisa, Italy

10. Division of Genetics and Epidemiology, The Institute of Cancer Research, Surrey, SM2 5NG, UK.

11. Division of Molecular Pathology, The Institute of Cancer Research, Surrey, SM2 5NG, UK.

12. National Centre of Tumor Diseases, Heidelberg, Germany

13. Institute of Human Genetics, University of Heidelberg, Heidelberg, Germany

14. Amyloidosis Research and Treatment Center, Foundation "Istituto di Ricovero e Cura a Carattere Scientifico (IRCCS) Policlinico San Matteo" and Department of Molecular Medicine, University of Pavia, Pavia, Italy;

15. National Amyloidosis Centre, University College London Medical School, Royal Free Hospital Campus, London, Rowland Hill Street, London NW32PF 
Words 2065.

$\underline{\text { E-mail addresses }}$

Kari Hemminki (K.Hemminki@dkfz.de)

Asta Försti (A.Foersti@dkfz.de)

Markus M. Nöthen (markus.noethen@uni-bonn.de)

Per Hoffmann (per.hoffmann@unibas.ch)

Karl-Heinz Jöckel <k-h.joeckel@uk-essen.de>

Hartmut Goldschmidt (Hartmut.Goldschmidt@med.uni-heidelberg.de)

Niels Weinhold <NWeinhold@uams.edu>

Gareth J. Morgan <GJMorgan@uams.edu>

Jolanta Nickel (jolinickel@gmx.de)

Miguel Inacio da Silva Filho ()

Iman Meziane ()

Chiara Campo ()

Stefano Landi<stefano.landi@unipi.it>

Anna Jauch < Anna.Jauch@med.uni-heidelberg.de>

Hartmut Goldschmidt <Hartmut.Goldschmidt@med.uni-heidelberg.de>

Ute Hegenbart (Ute.Hegenbart@ med.uni-heidelberg.de)

Stefanie Huhn (Stefanie.Huhn@med.uni-heidelberg.de)

Stefan Schönland (Stefan.Schoenland@med.uni-heidelberg.de)

Philip Hawkins (P.Hawkins@ucl.ac.uk)

Dorota Rowcieno (D.Rowcienco@ucl.ac.uk)

Ashu Wechalekar [a.wechalekar@ucl.ac.uk]

Paolo Milani (paolomilani.pv@gmail.com)

Giampaolo Merlini (gmerlini@unipv.it)

Giovanni Palladini (giovanni.palladini@unipv.it)

David Johnson <david.johnson@icr.ac.uk>;

Jonathan S. Mitchell <Jonathan.Mitchell@icr.ac.uk>;

Richard S Houlston <richard.houlston@icr.ac.uk> 


\section{ABSTRACT}

\section{INTRODUCTION}

Immunoglobulin light chain (AL) amyloidosis is a progressive plasma cell dyscrasia which is characterized by deposition of amyloid fibers derived from immunoglobulin light chain systemically in many organs ${ }^{1}$. Amyloidogenic light chains are secreted by clonal plasma cells and, because of the immunoglobulin variability, they are unique to each patient ${ }^{2,3}$. Characteristics of amyloids related to disease severity and sequelae, including the target organs where amyloids accumulate, such as the heart, kidney, liver and peripheral nerves ${ }^{3}$. Heart failure is usually the critical life-threatening condition; the median survival time depends on the extent of amyloid interference with the critical organ function and survival may range from some months to some years ${ }^{1,4}$. The incidence in AL amyloidosis is estimated to be somewhat over 3 per million ${ }^{5,6}$. Monoclonal gammopathy of undetermined significance (MGUS) is often the precursor disease for AL amyloidosis and the related disease multiple myeloma (MM) ${ }^{7}$. It has been reported that some 10 to $15 \%$ of multiple myeloma patients have AL amyloidosis ${ }^{8,9}$; conversely, some $10 \%$ of AL amyloidosis patients have MM at the time of diagnosis ${ }^{10}$. AL amyloidosis and MM share genetic risk loci and the 7 single nucleotide polymorphism (SNPs) initially described for MM were replicated in 443 AL amyloidosis patients with a nominal significance of $\mathrm{p}<0.05^{11}$.

We have recently characterized 10 putative genetic risk loci (at significance level of $<10^{-5}$ ) for AL amyloidosis using a genome-wide association study (GWAS) approach on a total of 1351 German, UK and Italian patients ${ }^{12}$. In the present study we carry out a systematic association study on the GWAS identified loci and the available clinical data including the affected organs and the type of serum immunoglobulin (Ig).

\section{METHODS}

The patient populations and GWAS analysis have been described elsewhere ${ }^{12}$. Shortly, the German amyloidosis patients (595 passed the GWAS quality control) were ascertained through the Amyloidosis Center at University Clinic Heidelberg. The UK samples (474) were obtained from the National Amyloidosis Centre, London and the Italian samples (282) came from the Amyloidosis Research and Treatment Center, 
Pavia. The diagnostic criteria used were as described ${ }^{13}$. DNA was genotyped using Illumina Human OmniExpress-12 v1.0 and related arrays. Local control populations included 2,107 Germans, 5637 Britons 465 Italians.

A total of 9 clinical profiles were selected among organ involvement (kidney, heart, heart \& kidney and liver, irrespective of whether other organs were involved) and Ig profiles (IgG with intact Ig, $\lambda$ any, $\kappa$ any, $\lambda / \kappa$ light chain only (LCO), and $\lambda \mathrm{LCO})$.

Analysis of the GWAS data was performed using imputed data as described ${ }^{12}$. All SNPs having a minor allele frequency $(\mathrm{MAF})<1 \%$ were excluded. The association test between imputed SNPs and AL amyloidosis was performed in SNPTESTv2.5. The three data sets were combined in meta-analysis and heterogeneity was assessed by the $\mathrm{I}^{2}$ statistic (interpreted as low $<0.25$, moderate 0.50 and high $>0.75$ ). Genomic locations are given in NCBI Build 37/UCSC hg19 coordinates. For genome-wide significance, a limit of $\mathrm{p}<5 \times 10^{-8}$ was used.

In order to test homogeneity of the results between AL amyloidosis and MM ASSET analysis was performed 14. This method explores all possible subsets for negative, positive, or null associations, identifying the subset with the strongest association signal; it also accounts for the multiple tests required by the subset analysis.

To investigate chromatin state segmentation profiles (ChromHMM) and 3-dimentional interactions (Hi-C) at risk loci we made use of the ENCODE project data on cell lines, including lymphoblastoid cells (GM12878). We also used HaploReg v4.1 (www.broadinstitute.org/mammals/haploreg) to evaluate the regulatory nature and the possible functional effects of SNPs and their proxies $r^{2} \geq 0.8$, or $0.95^{15}$. All relevant data available in HaploReg were considered in the SNP search but when multiple cell types were listed data on hematologic cell types were reported. Association profiles were visualized using the Locuszoom ${ }^{16}$ in conjunction with the UCSC genome browser ${ }^{17}$. Z score calculated for SNPs as log OR divided by standard error $^{18}$.

\section{RESULTS}


We selected 9 clinical profiles for a specific analysis of GWAS data (Table 1). Among Ig related profiles, $\lambda$ any (with or without heavy chains) was the most common one, found in 930 patients. $\lambda / \kappa$ LCO was found in 535 patients. Kidney and heart profiles were the largest organ profiles, including over 800 patients each, and liver profile was the smallest with only 194 patients. The median diagnostic ages differed minimally, from 62 to 66 years. The male-female ratio was 1.37 overall and it did not appreciably differ between the profiles. In the bottom of Table 1 data on the MM cohorts are given.

\section{Association analysis and comparison with MM}

We carried out a systematic association analysis of each of the 9 clinical profiles against controls in each of the 3 cohorts. Manhattan plots are shown for joint analysis in 4 clinical profiles with genome-wide associations. In the liver profile a genome-wide association, based on imputed SNPs, was noted in chromosome 11 but it had a MAF of $1 \%$; thus few individuals had the variant allele and the association was considered no further.

Among Ig profiles, the $\lambda / \kappa$ LCO and the $\lambda$ LCO profiles showed a strong association with SNP rs9344 (Table 2). The OR for rs 9344 OR in the $\lambda / \kappa$ LCO profile was $1.62\left(\mathrm{p}=1.99 \times 10^{-12}\right)$ and in the $\lambda$ LCO profile it was $1.70\left(\mathrm{p}=1.29 \times 10^{-11}\right)$. The weakest association was noted for the $\operatorname{IgG}$ profile $\left(1.20,9.69 \times 10^{-3}\right)$, with nonoverlapping 95\%CIs to the LCO profiles. For overall AL amyloidosis, the OR was 1.35 and for MM it was 1.06, as reported earlier ${ }^{12}$. Z-scores are also listed because they will be used in figures to be shown later.

For the IgG profile, rs10507419 reached genome-wide significance with an OR of 1.49 and p-value of $5.63 \times 10^{-8}$. The two subgroups $\operatorname{IgG} \lambda$ and $\operatorname{IgG} \kappa$ showed similar ORs (1.57 and 1.51, respectively) and $\operatorname{IgG} \lambda$ reached genome-wide significance of $2.90 \times 10^{-8}$ (Table 3). ORs of profiles $\lambda / \kappa \operatorname{LCO}(0.90), \lambda$ LCO (0.91), liver (0.98) and $\kappa$ any (1.00) differed significantly (non-overlapping 95\%Cs) from the IgG profile. Among MM subtypes, the OR for IgG MM was 1.01 while the ORs for both $\operatorname{IgG} \lambda$ and $\operatorname{IgG} \kappa$ were 1.00 .

Genome-wide association was found for SNP rs6752376 in the heart \& kidney profile (OR 1.54, p=2.88 x 10

${ }^{8}$ ) (Table 4). The profiles for kidney and heart only reached ORs of 1.24 and 1.27, respectively. ORs for liver 
(0.98) and $\kappa$ any (1.04) profiles differed significantly from the heart \& kidney profile. The OR for MM was 1.00 .

The liver profile rs7820212 reached genome-wide significance even with a small patient number (194) (OR 1.86, $\mathrm{p}=1.86 \times 10^{-8}$ ) (Table 5). ORs of all other clinical profiles differed significantly from the liver profile. The OR for AL amyloidosis overall was 1.07 and for MM it was 1.04.

Of note, there was no or at most moderate heterogeneity for any genome-wide significant associations in Tables 2 to 5 between the 3 AL amyloidosis cohorts as indicated by $\mathrm{I}^{2}$. The ORs of the significant associations did not change when stratified for age and sex.

We assessed the associations of the previously described 10 putative candidate SNPs from the combined AL amyloidosis cohorts with each of the 9 profiles ${ }^{12}$. With the exception of SNP rs9344 (Table 2) no other SNP associated specifically with AL amyloidosis defined by a clinical profile.

\section{Biological interference}

Regional plots of association are shown in Fig. 2 for the genome-wide significant SNPs in 4 clinical profiles. For the $\lambda / \kappa$ LCO profile, rs9344 on chromosome 11q13.3 maps to a splice site in the cyclin D1 gene as shown previously (Fig. 2A) ${ }^{12}$. For the IgG profile, SNP rs10507419 on chromosome 13q13.2 maps within the LINC00457 gene (long intergenic non-protein coding RNA 457) of unknown function and resides $330 \mathrm{~kb}$ 5' of NBEA (neurobeachin) (Fig, 2B). ENCODE Hi-C data are lacking for rs10507419 but data are available for the linked SNP $\left(\mathrm{r}^{2}=1.00\right) \mathrm{rs} 9529341,1 \mathrm{~kb}$ away, showing long-range association within the NBEA gene (Supplementary Fig. 1, not included). The SNP changes motif for transcription factor Pax-4. STEFAN: WE NEED DATA ON 13q deletion for this point.

The heart \& kidney profile risk SNP rs6752376 on chromosome 2p25.2 is located between two RNA genes, $63 \mathrm{~kb}$ from LINC01247 (long intergenic non-protein coding RNA 1247) and $9.9 \mathrm{~kb} 3$ ' of ACO17053.1 (not shown in Fig. 2C); functions of both of these are unknown. rs6752376 is a moderate expression quantitative 
trait locus (eQTL) $\left(3.7 \times 10^{-5}\right)$ to human metabolic profile relating to serum concentration of pantothenate ${ }^{19}$. According to HaploReg the SNP alters motifs for 3 transcription factors, Nkx2, Nkx3, PLZF. Liver profile SNP rs7820212 on chromosome 8q11.23 maps 28kb 3' of FAM150A (family with sequence similarity 150 member A). $116 \mathrm{~kb}$ away is the locus for RB1CC1 (RB1 inducible coiled-coil 1). The SNP changes motif for transcription factor CEBPB.

\section{DISCUSSION}

The recent GWAS on these 3 AL amyloidosis cohorts reported 4 SNPs reaching (or almost reaching) a genome-wide significance ${ }^{12}$. With the exception of the most significant SNP, rs9344, none of the other 3 were associated with the defined 9 clinical profiles, probably because of decreased patient numbers. Interestingly, 3 completely new profile-specific genetic loci were identified with homogeneous results from the 3 cohorts. Independent associations of rs9344 with the two LCO profiles and of rs10507419 with the two IgG profiles show internal consistency.

The preferential association of rs9344 with LCO profiles could possibly be explained by the association of this SNP with translocation $(11 ; 14)$ and the resulting disturbance of IgH production in AL amyloidosis and $\mathrm{MM}^{12,20,21}$. However no light chain excess has been reported in $\mathrm{t}(11 ; 14) \mathrm{AL}$ amyloidosis ${ }^{22,23}$. Data on MM cell line have suggested that compromised production of $\operatorname{IgH}$ leads to excess production of free light chains ${ }^{24}$. How rs9344 could interfere with $\mathrm{IgH}$ production independent of $\mathrm{t}(11 ; 14)$ remains enigmatic. Risk allele $\mathrm{G}$ at the splice site of cyclin D1 encodes a full length cyclin D1 which has many functions, including involvement in double-strand repair with RAD51, BRCA1 and BRCA2 and thus a possible interference with the class switch recombination for $I g H^{25,26}$. Curiously, while the LCO profiles were strongly associated with rs9344, the weakest association was noted for the IgG profile. Conversely, rs10507419 was strongly associated with the IgG profiles while weakly opposite associations were found with this SNP and the LCO profiles. rs10507419 on chromosome 13q13.2 maps close to the NBEA locus (13q13.3) which is a fragile site causing deletion of the telomeric end of chromosome 13q in patients with MM, MGUS and AL-amyloidosis 22, 27-29. We found in Hi-C data that rs10507419 shows long-range association with the NBEA locus.

Occasionally NBEA is fused with the tip of chromosome $8 \mathrm{q} 24$ containing $P V T 1^{30}$. The translocation may interfere with expression of $R B 1$ which is located at $13 q 14.2^{29}$. 
The possible functions of rs6752378 SNP associated with heart \& kidney profile are unknown as are those for the adjacent RNA genes LINC01247 and ACO17053.1. The SNP may influence serum concentration of pantothenate but how this might be related to the heart \& kidney profile remain unknown ${ }^{19}$. Liver profile SNP rs7820212 on chromosome 8q11.23 maps close to FAM150A, which is a ligand for receptor tyrosine kinases leukocyte tyrosine kinase (LTK) and anaplastic lymphoma kinase (ALK). These belong to the insulin receptor superfamily, and their aberrant activation has been described in many cancers, such as non-small lung cancer and neuroblastoma in which $A L K$ mutations are common ${ }^{31,32}$. Fusion genes of $A L K$ are often found in lymphomas with resulting downstream activation of the Ras/Raf/MEK/ERK pathway ${ }^{33}$. rs7820212 is adjacent to the $R B 1 C C 1$ gene which encodes a protein interacting with pathways involved in regulation of cell growth, proliferation, apoptosis, autophagy, and cell migration ${ }^{34,35}$. It has tumor suppressor properties

in enhancing RB1 (retinoblastoma 1) gene expression in cancer cells and promoting senescence ${ }^{36}$. The SNP changes the binding motif for CEBPB, which is an important transcription factor regulating the expression of genes involved in immune and inflammatory responses. CEBPB may regulate osteoclast activity in MM and through redundant functions with CEBPA it may be involved in multiple cellular processes in hematopoietic cells ${ }^{37,38}$.

In conclusion, 4 SNPs reached genome-wide associations in clinical profile-specific AL amyloidosis. While the associations were internally consistent and homogeneous between the 3 cohorts the underlying mechanisms remain speculative but tangible. For rs9344 the preference for LCO amyloidosis is another lead to mechanistic understanding.

\section{CONFLICT OF INTEREST STATEMENT}

The authors declare no competing financial interests.

\section{ACKNOWLEDGEMENTS}

Funding was provided by the German Cancer Aid, the Harald Huppert Foundations, The German Federal Ministry of Education and Research (eMed, Cliommics 01ZX1309B), the Multiple Myeloma Research Foundation, the Heinz Nixdorf Foundation (Germany), the Ministerium für Innovation, Wissenschaft und 
Forschung des Landes Nordrhein-Westfalen and the Faculty of Medicine University Duisburg-Essen. This study made use of genotyping data on the 1958 Birth Cohort generated by the Wellcome Trust Sanger Institute (http://www.wtccc.org.uk).

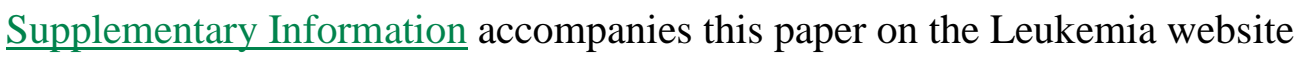

\section{REFERENCES}

1. Merlini G, Seldin DC, Gertz MA. Amyloidosis: pathogenesis and new therapeutic options. J Clin Oncol 2011; 29: 1924-1933.

2. Ramirez-Alvarado M. Amyloid formation in light chain amyloidosis. Current topics in medicinal chemistry 2012; 12(22): 2523-2533.

3. $\quad$ Blancas-Mejia LM, Ramirez-Alvarado M. Systemic amyloidoses. Annual review of biochemistry 2013; 82: 745-774.

4. Gertz M. Immunoglobin light chain amyloidosis: 2011 update on diagnosis, risk-stratification, and management Am J Hematol 2011; 86: 181-186.

5. Hemminki K, Li X, Forsti A, Sundquist J, Sundquist K. Incidence and survival in non-hereditary amyloidosis in Sweden. BMC Public Health 2012; 12: 974.

6. Pinney JH, Smith CJ, Taube JB, Lachmann HJ, Venner CP, Gibbs SD, et al. Systemic amyloidosis in England: an epidemiological study. Br J Haematol 2013; 161: 525-532.

7. Kyle RA, Durie BG, Rajkumar SV, Landgren O, Blade J, Merlini G, et al. Monoclonal gammopathy of undetermined significance (MGUS) and smoldering (asymptomatic) multiple myeloma: IMWG consensus perspectives risk factors for progression and guidelines for monitoring and management. Leukemia 2010 Jun; 24(6): 1121-1127.

8. $\quad$ Merlini G, Bellotti V. Molecular mechanisms of amyloidosis. N Engl J Med 2003; 349: 583-596.

9. Kyle RA, Rajkumar SV. Epidemiology of the plasma-cell disorders. Best Pract Res Clin Haematol 2007; 20: $637-664$.

10. Siragusa S, Morice W, Gertz MA, Kyle RA, Greipp PR, Lust JA, et al. Asymptomatic immunoglobulin light chain amyloidosis (AL) at the time of diagnostic bone marrow biopsy in newly diagnosed patients with multiple myeloma and smoldering myeloma. A series of 144 cases and a review of the literature. Ann Hematol 2011; 90: 101-106. 
11. Weinhold N, Forsti A, da Silva Filho MI, Nickel J, Campo C, Hoffmann P, et al. Immunoglobulin lightchain amyloidosis shares genetic susceptibility with multiple myeloma. Leukemia 2014 Jul 3; 28: 2254-2256.

12. da Silva Filho MI, Försti A, Weinhold N, Meziane I, Campo C, Huhn S, et al. Genome-wide association study of immunoglobulin light chain amyloidosis in three patient cohorts: comparison to myeloma. . Leukemia 2017.

13. Schonland SO, Hegenbart U, Bochtler T, Mangatter A, Hansberg M, Ho AD, et al. Immunohistochemistry in the classification of systemic forms of amyloidosis: a systematic investigation of 117 patients. Blood 2012 Jan 12; 119(2): 488-493.

14. Bhattacharjee S, Rajaraman P, Jacobs KB, Wheeler WA, Melin BS, Hartge P, et al. A subset-based approach improves power and interpretation for the combined analysis of genetic association studies of heterogeneous traits. Am J Hum Genet 2012 May 4; 90(5): 821-835.

15. Ward LD, Kellis M. HaploReg: a resource for exploring chromatin states, conservation, and regulatory motif alterations within sets of genetically linked variants. Nucleic Acids Res 2012 Jan; 40(Database issue): D930934.

16. Pruim RJ, Welch RP, Sanna S, Teslovich TM, Chines PS, Gliedt TP, et al. LocusZoom: regional visualization of genome-wide association scan results. Bioinformatics 2010; 26: 2336-2337.

17. Speir ML, Zweig AS, Rosenbloom KR, Raney BJ, Paten B, Nejad P, et al. The UCSC Genome Browser database: 2016 update. Nucleic Acids Res 2016 Jan 04; 44(D1): D717-725.

18. Li YR, Li J, Zhao SD, Bradfield JP, Mentch FD, Maggadottir SM, et al. Meta-analysis of shared genetic architecture across ten pediatric autoimmune diseases. Nat Med 2015 Sep; 21(9): 1018-1027.

19. Suhre K, Shin SY, Petersen AK, Mohney RP, Meredith D, Wagele B, et al. Human metabolic individuality in biomedical and pharmaceutical research. Nature 2011 Aug 31; 477(7362): 54-60.

20. Kumar S, Zhang L, Dispenzieri A, Van Wier S, Katzmann JA, Snyder M, et al. Relationship between elevated immunoglobulin free light chain and the presence of $\operatorname{IgH}$ translocations in multiple myeloma. Leukemia 2010 Aug; 24(8): 1498-1505.

21. Weinhold N, Johnson DC, Chubb D, Chen B, Försti A, Hosking FJ, et al. The CCND1 G870A polymorphism is a risk factor for $\mathrm{t}(11 ; 14)(\mathrm{q} 13 ; \mathrm{q} 32)$ multiple myeloma Nature Genet 2013; 45: 522-525.

22. Bochtler T, Hegenbart U, Cremer FW, Heiss C, Benner A, Hose D, et al. Evaluation of the cytogenetic aberration pattern in amyloid light chain amyloidosis as compared with monoclonal gammopathy of undetermined significance reveals common pathways of karyotypic instability. Blood 2008; 111: 4700-4705.

23. Muchtar E, Dispenzieri A, Kumar SK, Ketterling RP, Dingli D, Lacy MQ, et al. Interphase fluorescence in situ hybridization in untreated AL amyloidosis has an independent prognostic impact by abnormality type and treatment category. Leukemia 2016 Dec 16. 
24. Magrangeas F, Cormier ML, Descamps G, Gouy N, Lode L, Mellerin MP, et al. Light-chain only multiple myeloma is due to the absence of functional (productive) rearrangement of the IgH gene at the DNA level. Blood 2004 May 15; 103(10): 3869-3875.

25. Gostissa M, Alt FW, Chiarle R. Mechanisms that promote and suppress chromosomal translocations in lymphocytes. Annu Rev Immunol 2011; 29: 319-350.

26. Pestell RG. New Roles of Cyclin D1. The American Journal of Pathology 2013 7//; 183(1): 3-9.

27. Bochtler T, Hegenbart U, Heiss C, Benner A, Moos M, Seckinger A, et al. Hyperdiploidy is less frequent in AL amyloidosis compared with monoclonal gammopathy of undetermined significance and inversely associated with translocation t(11;14). Blood 2011 Apr 7; 117(14): 3809-3815.

28. Bochtler T, Hegenbart U, Kunz C, Benner A, Kimmich C, Seckinger A, et al. Prognostic impact of cytogenetic aberrations in AL amyloidosis patients after high-dose melphalan: a long-term follow-up study. Blood 2016 Jul 28; 128(4): 594-602.

29. O'Neal J, Gao F, Hassan A, Monahan R, Barrios S, Kilimann MW, et al. Neurobeachin (NBEA) is a target of recurrent interstitial deletions at 13q13 in patients with MGUS and multiple myeloma. Experimental hematology 2009 Feb; 37(2): 234-244.

30. Nagoshi H, Taki T, Hanamura I, Nitta M, Otsuki T, Nishida K, et al. Frequent PVT1 rearrangement and novel chimeric genes PVT1-NBEA and PVT1-WWOX occur in multiple myeloma with 8q24 abnormality. Cancer Res 2012 Oct 1; 72(19): 4954-4962.

31. Guan J, Umapathy G, Yamazaki Y, Wolfstetter G, Mendoza P, Pfeifer K, et al. FAM150A and FAM150B are activating ligands for anaplastic lymphoma kinase. eLife 2015 Sep 29; 4: e09811.

32. Reshetnyak AV, Murray PB, Shi X, Mo ES, Mohanty J, Tome F, et al. Augmentor alpha and beta (FAM150) are ligands of the receptor tyrosine kinases ALK and LTK: Hierarchy and specificity of ligand-receptor interactions. Proc Natl Acad Sci U S A 2015 Dec 29; 112(52): 15862-15867.

33. Roskoski R, Jr. Anaplastic lymphoma kinase (ALK): structure, oncogenic activation, and pharmacological inhibition. Pharmacological research 2013 Feb; 68(1): 68-94.

34. Pietrocola F, Izzo V, Niso-Santano M, Vacchelli E, Galluzzi L, Maiuri MC, et al. Regulation of autophagy by stress-responsive transcription factors. Semin Cancer Biol 2013 Oct; 23(5): 310-322.

35. Lebovitz CB, Robertson AG, Goya R, Jones SJ, Morin RD, Marra MA, et al. Cross-cancer profiling of molecular alterations within the human autophagy interaction network. Autophagy 2015; 11(9): 1668-1687.

36. Suraneni MV, Moore JR, Zhang D, Badeaux M, Macaluso MD, DiGiovanni J, et al. Tumor-suppressive functions of 15-Lipoxygenase-2 and RB1CC1 in prostate cancer. Cell cycle (Georgetown, Tex) 2014; 13(11): 1798-1810. 
37. Fuchs O, Kostecka A, Provaznikova D, Krasna B, Brezinova J, Filkukova J, et al. Nature of frequent deletions in CEBPA. Blood cells, molecules \& diseases 2009 Nov-Dec; 43(3): 260-263.

38. Tucci M, Stucci S, Savonarola A, Ciavarella S, Cafforio P, Dammacco F, et al. Immature dendritic cells in multiple myeloma are prone to osteoclast-like differentiation through interleukin-17A stimulation. $\mathrm{Br} \mathrm{J} \mathrm{Haematol}$ 2013 Jun; 161(6): 821-831. 
Table 1. Number of AL amyloidosis and multiple myeloma patients according to clinical profiles

\begin{tabular}{|c|c|c|c|c|c|c|c|}
\hline \multicolumn{2}{|c|}{ Clinical profiles } & German & British $^{c}$ & Italian & Joined & $\begin{array}{l}\text { Median age } \\
\text { (range) in years }{ }^{a}\end{array}$ & Sex-ratio $^{b}$ \\
\hline \multicolumn{2}{|c|}{ Overall AL amyloidosis } & 562 & 410 & 257 & 1129 & $64(30-87)$ & $1.37: 1$ \\
\hline \multirow[t]{7}{*}{ Ig profiles ${ }^{c}$} & $\mathrm{IgG}$ & 194 & 157 & 96 & 447 & $66(30-87)$ & $1.19: 1$ \\
\hline & $\operatorname{IgG} \lambda$ & 160 & 116 & 69 & 345 & $66(30-87)$ & $1.16: 1$ \\
\hline & $\operatorname{IgG} \kappa$ & 34 & 24 & 27 & 85 & $66(40-85)$ & $1.30: 1$ \\
\hline & $\lambda$ any & 438 & 304 & 188 & 930 & $64(30-87)$ & $1.38: 1$ \\
\hline & $\kappa$ any & 122 & 74 & 69 & 265 & $65(38-87)$ & $1.28: 1$ \\
\hline & $\lambda / \kappa \mathrm{LCO}$ & 312 & 96 & 127 & 535 & $62(37-84)$ & $1.49: 1$ \\
\hline & $\lambda \mathrm{LCO}$ & 231 & 84 & 89 & 404 & $62(37-84)$ & $1.59: 1$ \\
\hline \multirow[t]{4}{*}{ Organ profiles } & Kidney & 358 & 320 & 166 & 844 & $64(30-87)$ & $1.30: 1$ \\
\hline & Heart & 396 & 239 & 200 & 835 & $64(34-87)$ & $1.44: 1$ \\
\hline & $\mathrm{HK}$ & 180 & 140 & 106 & 426 & $63(38-87)$ & $1.39: 1$ \\
\hline & Liver & 105 & 57 & 32 & 194 & $63(34-87)$ & $1.49: 1$ \\
\hline \multicolumn{2}{|c|}{ Overall multiple myeloma } & 1508 & 2282 & - & 3790 & $63(27-89)$ & $1.41: 1$ \\
\hline \multirow[t]{3}{*}{ Ig profiles } & IgG MM & 748 & - & - & - & $57(30-72)$ & $1.44: 1$ \\
\hline & $\operatorname{IgG} \lambda \mathrm{MM}$ & 200 & - & - & - & $58(33-72)$ & $1.17: 1$ \\
\hline & $\operatorname{IgG} \kappa \mathrm{MM}$ & 548 & - & - & - & $57(30-72)$ & $1.55: 1$ \\
\hline
\end{tabular}

a: Median age of the joined cohort.

b: Sex-ratio is calculated as male:female ratio for the joined cohort.

c: Data on some clinical profiles were missing in the British cohort. 
Table 2. Summary statistics for the $\lambda / \kappa$ LCO risk allele G of rs 9344 in clinical profiles

\begin{tabular}{|c|c|c|c|c|c|c|}
\hline Profiles & Number of cases & Odds ratio & $95 \% \mathrm{CI}^{\mathrm{a}}$ & $\mathrm{P}$-value ${ }^{\mathrm{b}}$ & $I^{2 \mathrm{c}}$ & Z-score \\
\hline Overall AL & 1129 & 1.35 & $1.23-1.48$ & $7.80 \times 10^{-11}$ & 0.36 & 6.51 \\
\hline IgG & 447 & 1.20 & $1.05-1.38$ & $9.69 \times 10^{-3}$ & 0.00 & 2.59 \\
\hline$\lambda$ any & 930 & 1.40 & $1.27-1.55$ & $9.28 \times 10^{-11}$ & 0.00 & 6.48 \\
\hline$\kappa$ any & 265 & 1.33 & $1.11-1.59$ & $2.03 \times 10^{-3}$ & 0.00 & 3.09 \\
\hline$\lambda / \kappa \mathrm{LCO}$ & 535 & 1.62 & $1.42-1.85$ & $1.99 \times 10^{-12}$ & 0.00 & 7.04 \\
\hline$\lambda \mathrm{LCO}$ & 404 & 1.70 & $1.46-1.98$ & $1.29 \times 10^{-11}$ & 0.00 & 6.77 \\
\hline Kidney & 844 & 1.34 & $1.20-1.48$ & $6.89 \times 10^{-8}$ & 0.20 & 5.40 \\
\hline Heart & 835 & 1.39 & 1.24-1.54 & $2.91 \times 10^{-9}$ & 0.49 & 5.94 \\
\hline HK & 426 & 1.31 & $1.14-1.52$ & $2.14 \times 10^{-4}$ & 0.38 & 3.70 \\
\hline Liver & 194 & 1.40 & $1.14-1.73$ & $1.63 \times 10^{-3}$ & 0.00 & 3.15 \\
\hline Overall MM & 3790 & 1.06 & $1.00-1.12$ & $4.00 \times 10^{-2}$ & 0.61 & 2.09 \\
\hline
\end{tabular}

${ }^{\mathrm{a}} \mathrm{CI}$, confidence interval

${ }^{\mathrm{b}} \mathrm{P}$-value based on the meta-analysis of the three patient cohorts in AL amyloidosis, and two patient cohorts in multiple myeloma

${ }^{\mathrm{c}} I^{2}$ proportion of total variance due to heterogeneity

Genome-wide significant associations are indicated in bold 
Table 3. Summary statistics for the IgG profile risk allele A of rs10507419 of in clinical profiles

\begin{tabular}{|c|c|c|c|c|c|c|}
\hline Profiles & $\mathrm{N}$ cases & Odds ratio & $95 \% \mathrm{CI}^{\mathrm{a}}$ & P-value ${ }^{b}$ & $I^{2 \mathrm{c}}$ & Z-score \\
\hline Overall AL & 1129 & 1.13 & $1.03-1.25$ & $1.15 \times 10^{-2}$ & 0.00 & 2.53 \\
\hline IgG & 447 & 1.49 & $1.29-1.72$ & $5.63 \times 10^{-8}$ & 0.49 & 5.43 \\
\hline $\operatorname{IgG} \lambda$ & 345 & 1.57 & $1.34-1.85$ & $2.90 \times 10^{-8}$ & 0.42 & 5.55 \\
\hline $\operatorname{IgG} \kappa$ & 85 & 1.51 & $1.21-1.89$ & $2.39 \times 10^{-4}$ & 0.67 & 3.68 \\
\hline$\lambda$ any & 930 & 1.18 & $1.06-1.32$ & $2.20 \times 10^{-3}$ & 0.00 & 3.06 \\
\hline$\kappa$ any & 265 & 1.00 & $0.82-1.22$ & $9.88 \times 10^{-1}$ & 0.00 & 0.02 \\
\hline$\lambda / \kappa \mathrm{LCO}$ & 535 & 0.90 & $0.78-1.04$ & $1.63 \times 10^{-1}$ & 0.00 & -1.39 \\
\hline$\lambda \mathrm{LCO}$ & 404 & 0.91 & $0.77-1.07$ & $2.46 \times 10^{-1}$ & 0.35 & -1.16 \\
\hline Kidney & 844 & 1.18 & $1.06-1.32$ & $2.69 \times 10^{-3}$ & 0.00 & 3.00 \\
\hline Heart & 835 & 1.16 & $1.04-1.30$ & $1.02 \times 10^{-2}$ & 0.00 & 2.57 \\
\hline $\mathrm{HK}$ & 426 & 1.33 & $1.15-1.55$ & $1.81 \times 10^{-4}$ & 0.38 & 3.75 \\
\hline Liver & 194 & 0.98 & $0.78-1.24$ & $9.00 \times 10^{-1}$ & 0.00 & -0.13 \\
\hline Overall MM & 3790 & 1.06 & $1.00-1.13$ & $4.47 \times 10^{-2}$ & 0.03 & 2.00 \\
\hline $\operatorname{IgG~MM~}$ & 748 & 1.01 & $0.88-1.15$ & $9.35 \times 10^{-1}$ & - & 0.08 \\
\hline $\operatorname{IgG} \lambda \mathrm{MM}$ & 200 & 1.00 & $0.80-1.26$ & $9.56 \times 10^{-1}$ & - & 0.06 \\
\hline $\operatorname{IgG} \kappa \mathrm{MM}$ & 548 & 1.00 & $0.86-1.16$ & $9.40 \times 10^{-1}$ & - & 0.07 \\
\hline
\end{tabular}

${ }^{\mathrm{a} C I}$, confidence interval

${ }^{\mathrm{b}} \mathrm{P}$-value based on the meta-analysis of three patient cohorts in AL amyloidosis, and two patient cohc in multiple myeloma; the IgG profiles of MM are based on only German cohort ${ }^{\mathrm{c}} I^{2}$ proportion of total variance due to heterogeneity

Genome-wide significant associations are indicated in bold 
Table 4. Summary statistics for the HK profile risk allele T of rs6752376 in clinical profiles

\begin{tabular}{lllllll} 
Profiles & Number of cases & Odds ratio & $95 \% \mathrm{CI}^{\mathrm{a}}$ & P-value $^{\mathrm{b}}$ & $I^{2 \mathrm{c}}$ & Z-score \\
\hline Overall AL & 1129 & 1.17 & $1.06-1.28$ & $9.96 \times 10^{-4}$ & 0.75 & 3.29 \\
IgG & 447 & 1.20 & $1.04-1.39$ & $1.12 \times 10^{-2}$ & 0.33 & 2.54 \\
$\lambda$ any & 930 & 1.24 & $1.12-1.38$ & $5.20 \times 10^{-5}$ & 0.67 & 4.05 \\
$\kappa$ any & 265 & 1.04 & $0.87-1.25$ & $6.59 \times 10^{-1}$ & 0.10 & 0.44 \\
$\lambda / \kappa$ LCO & 535 & 1.20 & $1.05-1.37$ & $7.78 \times 10^{-3}$ & 0.46 & 2.66 \\
$\lambda$ LCO & 404 & 1.25 & $1.08-1.46$ & $3.52 \times 10^{-3}$ & 0.54 & 2.92 \\
Kidney & 844 & 1.24 & $1.11-1.38$ & $8.62 \times 10^{-5}$ & 0.71 & 3.93 \\
Heart & 835 & 1.27 & $1.14-1.42$ & $1.50 \times 10^{-5}$ & 0.30 & 4.31 \\
HK & $\mathbf{4 2 6}$ & $\mathbf{1 . 5 4}$ & $\mathbf{1 . 3 2 - 1 . 7 9}$ & $\mathbf{2 . 8 8 \times 1 0 ^ { - 8 }}$ & $\mathbf{0 . 0 7}$ & 5.55 \\
Liver & 194 & 0.98 & $0.80-1.21$ & $8.86 \times 10^{-1}$ & 0.00 & -0.14 \\
\hline Overall MM & 3790 & 1.00 & $0.94-1.06$ & $9.25 \times 10^{-1}$ & 0.37 & -0.09 \\
\hline
\end{tabular}

${ }^{a} \mathrm{CI}$, confidence interval

${ }^{\mathrm{b}} \mathrm{P}$-value based on the meta-analysis of three patient cohorts in $\mathrm{AL}$ amyloidosis, and two patient cohorts in multiple myeloma

${ }^{c} I^{2}$ proportion of total variance due to heterogeneity

Genome-wide significant associations are indicated in bold 
Table 5. Summary statistics for the liver profile risk allele A of rs7820212 in clinical profiles

\begin{tabular}{lllllll} 
Profiles & Number of cases & Odds ratio & $95 \% \mathrm{CI}^{\mathrm{a}}$ & $\mathrm{P}^{\text {-value }}{ }^{\mathrm{b}}$ & $I^{2 \mathrm{c}}$ & Z-score \\
\hline Overall AL & 1129 & 1.07 & $0.98-1.17$ & $1.40 \times 10^{-1}$ & 0.19 & 1.48 \\
IgG & 447 & 1.00 & $0.87-1.15$ & $9.63 \times 10^{-1}$ & 0.00 & 0.05 \\
$\lambda$ any & 930 & 1.10 & $0.99-1.21$ & $6.32 \times 10^{-2}$ & 0.52 & 1.86 \\
$\kappa$ any & 265 & 0.97 & $0.81-1.16$ & $7.49 \times 10^{-1}$ & 0.05 & -0.32 \\
$\lambda / \kappa$ LCO & 535 & 1.13 & $0.99-1.29$ & $6.67 \times 10^{-2}$ & 0.26 & 1.83 \\
$\lambda$ LCO & 404 & 1.14 & $0.98-1.32$ & $8.30 \times 10^{-2}$ & 0.61 & 1.73 \\
Kidney & 844 & 1.10 & $0.99-1.22$ & $8.33 \times 10^{-2}$ & 0.36 & 1.73 \\
Heart & 835 & 1.09 & $0.98-1.21$ & $1.07 \times 10^{-1}$ & 0.00 & 1.61 \\
HK & 426 & 1.02 & $0.88-1.17$ & $8.04 \times 10^{-1}$ & 0.06 & 0.25 \\
Liver & $\mathbf{1 9 4}$ & $\mathbf{1 . 8 6}$ & $\mathbf{1 . 5 0 - 2 . 3 1}$ & $\mathbf{1 . 8 6 \times 1 0 ^ { - 8 }}$ & $\mathbf{0 . 0 4}$ & 5.63 \\
\hline Overall MM & 3790 & 1.04 & $0.98-1.10$ & $1.81 \times 10^{-1}$ & 0.00 & 1.34 \\
\hline
\end{tabular}

${ }^{a} \mathrm{CI}$, confidence interval

${ }^{\mathrm{b}} \mathrm{P}$-value based on the meta-analysis of three patient cohorts in AL amyloidosis, and two patient cohorts in multiple myeloma

${ }^{c} I^{2}$ proportion of total variance due to heterogeneity

Genome-wide significant associations are indicated in bold 


\section{LEGENDS TO FIGURES}

Figure 1. Manhattan plots of association analysis for AL amyloidosis clinical profiles with genome-wide significant results. A) $\lambda / \kappa$ LCO profile; B) IgG profile; C) heart \& kidney profile; D) liver profile. The $\mathrm{x}$-axis shows the chromosomal position and the $\mathrm{y}$-axis is the significance ($\log 10 \mathrm{P} ; 2$-tailed) of association derived by logistic regression. The red line shows the genomewide significance level $\left(5 \times 10^{-8}\right)$ and the blue line shows suggestive significance level $\left(1 \times 10^{-5}\right)$. The significant/top SNPs are labeled.

Figure 2. Regional association plots showing the significant/top SNPs in the four AL amyloidosis clinical profiles. A) $\lambda / \kappa$ LCO profile; B) IgG profile; C) heart \& kidney profile; D) liver profile. The xaxis shows the chromosomal position as $\mathrm{Mb}$ and the mapped genes annotated from the UCSC genome browser. The y-axis shows the significance (- $\log 10 \mathrm{P} ; 2$-tailed) on the left and recombination rates (light blue lines) on the right. The reference SNP is labeled and colored purple, the rest of the SNPs are colored based on their $\mathrm{r}^{2}$ to the reference SNP, on a shown scale, based on pairwise $\mathrm{r}^{2}$ values from HapMap CEU. Square-shaped SNP symbols represent genotyped SNPs and circle-shaped SNPs represent imputed ones.

Supplementary Figure : Z-diagrams

\section{Supplementary Figure : Hi-C}

Supplementary Figure : Forest plot for each SNP considering amyloidosis and multiple myeloma results. 